\title{
Risk Aversion and the Value of Risk to Life
}

\author{
Antoine Bommier* \\ Bertrand Villeneuve ${ }^{\dagger}$ \\ antoine.bommier@univ-tlse1.frｂertrand.villeneuve@ensae.fr
}

18 June 2009

\begin{abstract}
The standard literature on the value of life relies on Yaari's (1965) model, which includes an implicit assumption of risk neutrality with respect to life duration. To overpass this limitation, we extend the theory to a simple variety of nonadditively separable preferences. The enlargement we propose is relevant for the evaluation of life-saving programs: current practice, we estimate, puts too little weight on mortality risk reduction of the young. Our correction exceeds in magnitude that introduced by the switch from the notion of number of lives saved to the notion of years of life saved.

Keywords: Value of Statistical Life; Lifecycle Behavior; Cost-benefit Analysis; Life Insurance.
\end{abstract}

JEL: D61, D81, D91, I18, J17.

*Address: Toulouse School of Economics (CNRS, GREMAQ), 21 allée de Brienne, 31000 Toulouse, France.

$\dagger$ Address: CREST, Laboratoire de Finance Assurance, 15 boulevard Gabriel Péri, 92245 Malakoff cedex, France. Other affiliations: Université de Tours and CESifo, Munich. 


\title{
Risk Aversion and the Value of Risk to Life
}

\begin{abstract}
The standard literature on the value of life relies on Yaari's (1965) model, which includes an implicit assumption of risk neutrality with respect to life duration. To overpass this limitation, we extend the theory to a simple variety of nonadditively separable preferences. The enlargement we propose is relevant for the evaluation of life-saving programs: current practice, we estimate, puts too little weight on mortality risk reduction of the young. Our correction exceeds in magnitude that introduced by the switch from the notion of number of lives saved to the notion of years of life saved.
\end{abstract}

Keywords: Value of Statistical Life; Lifecycle Behavior; Cost-benefit Analysis; Life Insurance.

JEL: D61, D81, D91, I18, J17.

\section{Introduction}

Billions of dollars are spent every year on mortality reduction programs. Issues like the allocation of funds to medical research or prevention, the design of safety rules or the wording of environmental bills raise intense debate on the relevance of the choices made by governments and their agencies. For economists, the baseline is that alternative projects should be evaluated with objective criteria to avoid pure waste or dramatic underinvestment in less popular issues.

To back public decisions, some inquiry into individual valuation of life is indispensable. In practice, if we leave apart contingent valuation, the analysis of the wage-risk tradeoff is the major source of estimates of people's behavior with respect to risk to life. These surveys are primarily informative about industrial workers. Since public programs affect wider populations whose characteristics may vary considerably and given that the mortality changes considered are often beyond the range experienced by the reference sample, a theoretical support for the interpretation of the data is indispensable.

The choice of the structural life-cycle model that minimizes bias at estimation and extrapolation stages is capital. The standard approach uses additively separable life-cycle 
models. The intertemporal additivity assumption, which involves an implicit assumption of risk neutrality with respect to length of life is extremely constraining (Bommier 2006). Although this model has been severely criticized in other branches of literature, ${ }^{1}$ it remains an almost universal assumption for applied theory papers on the value of life. ${ }^{2}$

In this paper, we develop an alternative model, based on recursive von Neumann-Morgenstern utility functions, which relaxes the additivity assumption and thereby introduces what we shall call mortality risk aversion. ${ }^{3}$ Although this extension complicates intermediate calculations, practical difficulties are kept at a reasonable level: formulas for the value of statistical lives are almost as simple as those obtained with the standard additive model. There are therefore no technical difficulties for applying this novel approach to concrete issues. Above all, relaxing additivity warrants a significant gain in accuracy. As a proof of concept, we use empirical results on the wage-risk tradeoff to calibrate both the additive and nonadditive models. While the additive model proves unable to fit the data, the generalization proposed provides an excellent fit with reasonable estimated parameters.

To emphasize the importance of accounting for mortality risk aversion, we compare the benefits of (fictitious) life saving policies using different methods. The magnitude of the bias caused by the additive separability assumption appears to be uncomfortably big. The type of cost-benefit analysis that is currently recommended for life-saving programs is likely to be strongly biased in favor of the elderly if the decline of the VSL with age is underestimated. The correction we suggest could exceed in magnitude that introduced by the switch from the notion of number of lives saved to the notion of years of life saved.

Life insurance companies are primarily concerned with the costs of providing coverage.

\footnotetext{
${ }^{1}$ Even when mortality is not an issue, theoretical arguments underlined unpleasant consequences of the additive separability assumption (e.g. Richard 1975, Deaton 1974 and 1992, Epstein and Zin 1991). Moreover, the additive model's inability to fit intertemporal choice has been repeatedly underlined by empirical studies (Hayashi 1985, Muellbauer 1988, Browning 1991, and Carrasco, Labeaga and López-Salido 2005).

${ }^{2}$ See for example the recent contributions of Murphy an Topel (2006) and Hall and Jones (2007).

${ }^{3}$ It should be clear that the nonadditive model we use introduces a variety of risk aversion towards life length that is to be distinguished from financial risk aversion as in Eeckhoudt and Hammitt (2004) and Kaplow (2005). These papers discuss the impact of the curvature of the instantaneous utility function on the VSL. This issue matters particularly for understanding the income elasticity of the VSL Kaplow (2005). The pure effect of age is not investigated.
} 
These costs are directly related to the probabilities of the events covered, hence the importance of mortality tables. For policyholders however, the notion of complete or perfect insurance is not as clear as for loss insurance (where a simple dollar-for-dollar rule works pretty well). The structure of individual preferences matters to design contributions and benefits plans. Life insurance products are, empirically, highly diversified. This reflects the diversity of individual preferences and the diversity of insurer sophistication as to the understanding of these preferences.

The empirical wage-risk tradeoff allows us to test alternative theories of the lifecycle preferences. Potentially, this better understanding enables testing the efficiency of actual insurance policies and could help improve design, and thereby, market performance.

The two main applications we mention in this paper (longevity related public policy, life insurance design) are tightly connected. As longevity prospects evolve, and because this evolution is partly stochastic (i.e. revealed progressively as people age and, say, medical discoveries are made), insurers may want to introduce options in their products. These options could be additional contributions at predefined dates, changes in the indexing rule, cash surrender, etc. The value of these options and how they will be exercized would be better assessed with a better behavioral model. These considerations of course are programmatic and go beyond the contribution of this particular paper.

The structure of the paper is as follows: a theory is set up and analyzed, parameters based on empirical results are given, a prediction of optimal public choice is proposed for illustration. Section 2 positions our work in the recent related literature. Section 3 recalls the additive model, introduces more general preferences and characterizes mortality risk aversion. Section 4 shows the consequences of alternative models for the individual valuation of statistical lives. Using an available hedonic regression of the value of statistical life, Section 5 searches for the best fitting model and shows the performance of the nonadditive version. Section 6 contrasts quantitatively several evaluation procedures on typical life saving programs. 


\section{Related literature}

Most of the economic literature on the Value of Statistical Life (henceforth VSL) is based on a particular model whose standard version (e.g. Arthur 1981, Shepard and Zeckhauser 1984 or Rosen 1988) relies on elements developed in Yaari (1965). Several extensions have recently been suggested.

In Murphy and Topel (2006), health multiplies the instantaneous utility derived from the flow of consumption. Since health is assumed to be exogenous in that part of their paper assessing the gain from mortality risk reduction, their approach is equivalent to assuming that agents have additively separable utility functions whose (exogenous) discount function is not necessarily exponential. Hall and Jones (2007) also extend Yaari's model by introducing a health component in the utility function. Still, health being unobserved, they end up assuming in applications that it equals the inverse of the mortality rate. Though sensible, this amounts to assuming that instantaneous utility depends on mortality through a particular functional form. Ehrlich and Yin (2005) model a technology through which protection expenditures increase longevity; the authors also introduce a bequest motive.

The above contributions extended Yaari's model in several directions, but have in common that they all maintain the assumption of additive separability of preferences. It is precisely that later assumption that we shall relax. Our contribution is thus of a different nature: instead of incorporating additional variables to Yaari's model (such as health or bequest), we explore the potential of a less straightly structured specification. As we shall see, this provides different insights, especially on the speed at which VSL may or may not decline with age at old ages.

The effect of age on the VSL is controversial. ${ }^{4}$ Simple simulations of the original models exhibit either a decline with age, or an inverse U-shape. When careful calibration is achieved to match empirical consumption profiles, the inverse U-shape is generally found, with a rather slow decline at old ages. The above mentioned theoretical extensions of Murphy and Topel (2006) and Ehrlich and Yin (2005) tend to confirm this prediction. Empirical works, however,

\footnotetext{
${ }^{4}$ See the discussion in Aldy and Viscusi (2007) and the references to press articles therein.
} 
do not converge to a consensus on the relation between age and VSL. The hedonic regressions on wages in Aldy and Viscusi (2003) and Kniesner et al. (2006) also show an inverse U-shape relation between age and VSL, with a rather rapid decline of VSL at old ages. Other recent works (Alberini et al. 2004, Smith et al. 2004, Aldy and Viscusi, 2008), based either on contingent valuation or wage-risk tradeoffs, tend to minimize the significant decline that was apparent in previous estimates. The debate seems far from being closed. The present paper contributes to it by showing that when the assumption of additive separability of preferences is relaxed in order to account for mortality risk aversion, then a rapid decline of VSL at old ages becomes theoretically plausible.

\section{$3 \quad$ Lifetime preferences}

\subsection{Basic concepts and notation}

We define a life as the cross product of an infinite consumption profile $\mathbf{c}$ and a finite age at death $T$. For an individual of age $a$, a life $(\mathbf{c}, T)$ is an element of $\mathcal{L}_{a}$ with

$$
\mathcal{L}_{a}=C([a,+\infty[, \mathbb{R}) \times[a,+\infty[
$$

where $C([a,+\infty[, \mathbb{R})$ denotes the set of continuous functions mapping $[a,+\infty[$ into $\mathbb{R}$. Consumption at age $t$ is denoted by $c_{t}$. Note that consumption is not a priori constrained to equal zero for $t>T$, but this will have no importance since it will be assumed that agents do not care for consumption after death.

Lifetime being uncertain, modeling the tradeoff between mortality and consumption requires a theory of choice under risk. We apply the VNM expected utility framework on the space of lotteries (i.e. probability measures) over $\mathcal{L}_{a}$. To do so, one can define a utility function (or Bernoulli index) $U_{a}(\mathbf{c}, T)$ such that for any two probability measures $\eta, \eta^{\prime}$ on $\mathcal{L}_{a}$,

$$
\eta \succeq \eta^{\prime} \Leftrightarrow E_{\eta} U_{a} \geq E_{\eta^{\prime}} U_{a}
$$


where $\succeq$ denotes weak preference and $E_{\eta}$ (resp. $E_{\eta^{\prime}}$ ) is the expectation operator based on probability $\eta$ (resp. $\eta^{\prime}$ ).

We assume that individuals do not care for consumption after death, which amounts to posing $U_{a}(\mathbf{c}, T)=U_{a}\left(\mathbf{c}^{\prime}, T\right)$ for any two $\mathbf{c}, \mathbf{c}^{\prime}$ that are equal on $[a, T]$. This enables us to normalize $U_{a}$ so as to have $U_{a}(\mathbf{c}, a)=0, \forall \mathbf{c}$.

A probability measure over $\mathcal{L}_{a}$ for which the consumption profile $\mathbf{c}$ is predetermined and the uncertainty bears only on $T$ can be written as $\delta_{\mathbf{c}} \times m$ where $\delta_{\mathbf{c}}$ is a Dirac and $m$ is a probability measure over $[a,+\infty)$ describing the distribution of the age of death. We have

$$
E_{\delta_{\mathbf{c} \times m}} U_{a}=\int_{a}^{+\infty} U_{a}(\mathbf{c}, T) m(T) d T .
$$

This expected utility will be simply denoted by $E U_{a}$ in the rest of the paper.

The probability of being alive at age $T$, conditional on being alive at age $a$, is denoted

$$
s_{a}^{T} \equiv \exp \left(-\int_{a}^{T} \mu_{t} d t\right)=1-\int_{a}^{T} m(t) d t
$$

where the latter equality expresses survival in terms of mortality rates, $\mu_{t}$ being the hazard rate of death at age $t$.

We make two purely technical assumptions.

Assumption $1 \mu_{t}$ tends to infinity as $t$ tends to infinity.

Assumption $2 \mathbf{c}$ is bounded in the long run, i.e. there is an interval $\left[c_{\min }, c_{\max }\right]$ with $c_{\min }>$ 0 and $c_{\max }<+\infty$ on which $\mathbf{c}$ is supported after some arbitrary date.

Integration by parts yields

$$
E U_{a}=\left[-s_{a}^{T} U_{a}(\mathbf{c}, T)\right]_{a}^{+\infty}+\int_{a}^{+\infty} s_{a}^{T} \frac{\partial U_{a}(\mathbf{c}, T)}{\partial T} d T
$$

and eventually, using Assumptions 1 and $2\left(s_{a}^{T} \rightarrow 0\right.$ and $U_{a}$ is bounded as $\left.T \rightarrow+\infty\right)$ to 
evaluate the first term, we find

$$
E U_{a}=\int_{a}^{+\infty} s_{a}^{T} \frac{\partial U_{a}(\mathbf{c}, T)}{\partial T} d T
$$

The potential of the theory now depends on the assumptions that are made on $U_{a}(\mathbf{c}, T)$ or equivalently on $\frac{\partial U_{a}(\mathbf{c}, T)}{\partial T}$. We first come back on the common additive specification and highlight some of its properties. Then we suggest a more general form for $U_{a}(\mathbf{c}, T)$ on which our analysis will be based.

\subsection{The additive model}

The usual approach relies on elements developed in Yaari (1965). In this "additive model," preferences are time consistent, age and history independent and additively separable. An individual of age $a$ has preferences represented by the Bernoulli index

$$
U_{a}^{\text {add }}(\mathbf{c}, T)=\int_{a}^{T} u\left(c_{t}\right) e^{-\lambda(t-a)} d t
$$

where $u$ is a well-behaved instantaneous utility function and $\lambda$ is the subjective discount factor. In this case

$$
\frac{\partial U_{a}^{\text {add }}(\mathbf{c}, T)}{\partial T}=u\left(c_{T}\right) e^{-\lambda(T-a)}
$$

and the expected utility is

$$
E U_{a}^{\mathrm{add}}=\int_{a}^{+\infty} s_{a}^{T} u\left(c_{T}\right) e^{-\lambda(T-a)} d T
$$

We recognize the formulation in Yaari (1965).

A peculiar feature of this model is that $\frac{\partial U_{a}^{\text {add }}(\mathbf{c}, T)}{\partial T}$ is independent of past consumption. Said differently, the marginal utility of life is independent of how good (or bad) life has been in the past. ${ }^{5}$ If we parallel this with wealth preferences, this is akin to assuming that marginal

\footnotetext{
${ }^{5}$ Note that introducing a relation between marginal utility of life and past consumption would not necessarily require assuming that preferences are history dependent. See for example the case of recursive utilities
} 
utility of wealth is independent of wealth, i.e. that the decision maker is risk neutral. This point was first stressed by Broome (1993) who criticized the additive specification for relying on an implicit assumption of "risk neutrality over discounted QALY's", which he qualified as "surely implausible" (Broome, 1993, p.166). In a parallel line of argument, Bommier (2006) shows that assuming additive separability as in (7) is equivalent to assuming risk neutrality over life duration when considering consumption paths whose variations compensate for time preferences.

\subsection{The recursive model}

One could think of several tractable options with marginal utility of life depending on past consumption. The approach we follow preserves stationarity, one of the key properties of Yaari's specification. ${ }^{6}$ Basically, stationarity means that preferences are time consistent, independent of age and of history (see Epstein 1983, who extended Koopmans' 1965 definition of stationarity to the case of choice under uncertainty). In other words, people of different ages differ only with respect to their earning and mortality profiles. Bommier (2005) shows in particular that for agents that are sure to die (but who may not know when they will die), preferences are stationary if and only if they can be represented as

$$
U_{a}(\mathbf{c}, T)=\int_{a}^{T} u\left(c_{t}\right) \exp \left(-\int_{a}^{t} v\left(c_{\tau}\right) d \tau\right) d t
$$

This specification first appeared in the economic literature (in the case of immortal agents) in Uzawa (1969). As soon as we depart from the additive case, the meanings of $u$ and $v$ are not straightforward. Uzawa interpreted the integral $\int_{a}^{t} v\left(c_{\tau}\right) d \tau$ as an "accumulated rate of time preference". This extrapolation from the additive model is misleading: it suggests

considered in the following section.

${ }^{6}$ Another possibility is to allow changes in risk aversion à la Kihlstrom and Mirman (1974). This is pursued in Bommier (2006). Instead of taking the expected value of $U_{a}^{\text {add }}(\mathbf{c}, T)$, one uses $\Phi\left(U_{a}^{\text {add }}(\mathbf{c}, T)\right)$ where $\Phi$ is an increasing transformation. The drawback is that these preferences are not stationary, except in two cases: $\Phi$ is linear (the additive case) or $\Phi$ is exponential and $\lambda=0$ (the multiplicative model discussed later on in the present paper). 
that the rate of time discounting depends on past consumption whereas, due to their recursive form, preferences are indeed characterized by independence with respect to it. ${ }^{7} \mathrm{~A}$ rigorous approach involves starting from well defined local properties of individual preferences (marginal rates of substitution) and deriving proper concepts of time discounting and intertemporal elasticity of substitution, as will be done in Subsection 3.4.

Two special cases of the recursive model (10) must be highlighted at this stage. They are equally simple and the empirical part of this paper will show a clear difference (in favor of the second) in their abilities to fit data. The first one is simply the additive one (take $v(\cdot)=\lambda$, a constant). The second one is the multiplicative model in which $v(c)=k u(c), \forall c$, for some constant $k$; equation (10) can be integrated to give

$$
U_{a}^{\text {multi }}(\mathbf{c}, T)=\frac{1-\exp \left(-k \int_{a}^{T} u\left(c_{t}\right) d t\right)}{k}
$$

The term multiplicative refers to the fact that the exponentials of the instantaneous utilities multiply each other. Being a concave transformation of an additive utility function, this latter specification maintains the assumption of weak separability of preferences. Increasing $k$ amounts to increasing risk aversion in the sense of Kihlstrom and Mirman (1974). This specification is therefore particularly appropriate to illustrate the impact of risk aversion on the value of risk to life.

Under uncertain lifetime, the expected utility based on (10) is

$$
E U_{a}=\int_{a}^{+\infty} s_{a}^{t} u\left(c_{t}\right) \exp \left(-\int_{a}^{t} v\left(c_{\tau}\right) d \tau\right) d t
$$

This paper will not discuss the consequences, for given mortality, of recursive preferences on the intertemporal allocation of wealth. ${ }^{8}$ We focus instead on issues related to endogenous

\footnotetext{
${ }^{7}$ To see recursivity, remark that for all $a, b, T$ such that $a \leq b \leq T$

$$
U_{a}(\mathbf{c}, T)=\int_{a}^{b} u\left(c_{t}\right) \exp \left(-\int_{a}^{t} v\left(c_{\tau}\right) d \tau\right) d t+\exp \left(-\int_{a}^{b} v\left(c_{\tau}\right) d \tau\right) \cdot U_{b}(\mathbf{c}, T)
$$

${ }^{8}$ Consumption smoothing with this kind of preferences is discussed at length in Bommier (2005) for
} 
mortality choices, a typical example of which being the wage-risk tradeoff. For this purpose, we need a few general concepts.

\subsection{Local properties}

The first concept expresses how individuals trade off present and future consumptions: ${ }^{9}$

Definition 1 (RD) The mortality adjusted rate of time discounting at age $t$ is

$$
R D(\mathbf{c}, t) \equiv-\left.\frac{d}{d t} \log \left(\frac{1}{s_{a}^{t}} \cdot \frac{\partial E U_{a}}{\partial c_{t}}\right)\right|_{\dot{c}_{t}=0}
$$

In absence of mortality at age $t$ (i.e. if $s_{a}^{t}$ were constant around $t$ ), $\mathrm{RD}(\mathbf{c}, t)$ would be the rate of time discounting in continuous time defined in Epstein (1987). The correction $1 / s_{a}^{t}$ simply neutralizes the uncertainty effect that mortality risk has on consumption (consumption is contingent on survival). With the recursive model, calculations yield

$$
\mathrm{RD}(\mathbf{c}, t)=\frac{v\left(c_{t}\right) u^{\prime}\left(c_{t}\right)-v^{\prime}\left(c_{t}\right)\left(u\left(c_{t}\right)-\mu_{t} E U_{t}\right)}{u^{\prime}\left(c_{t}\right)-v^{\prime}\left(c_{t}\right) E U_{t}},
$$

where $E U_{t}$ is defined in (12). Although the definition of $\mathrm{RD}(\mathbf{c}, t)$ is conditional on $a$, the current age of the individual, $\mathrm{RD}(\mathbf{c}, t)$ only depends on consumption and mortality at ages greater than or equal to $t$. This is a consequence of history independence and time consistency: a 20 year old individual and a 50 year old individual anticipate the same value for the rate of discount of consumption at age 60 . The indexes defined below exhibit similar properties of independence from the past.

The second concept, intertemporal elasticity of substitution, is defined with continuous time as the limit of the direct elasticity of substitution (as defined in McFadden, 1963)

nonconstant $v$. A causal link between mortality (as a risk) and apparent impatience is put forward. In particular, with the multiplicative model which rules out pure time preference, sizable impatience can be calculated even with small mortality rates.

${ }^{9}$ Because of our continuous time modeling, we use Volterra derivatives. They measure utility changes when consumption (or mortality) varies by an infinitesimal value during an infinitesimally short lapse of time. For example $\frac{\partial U_{a}}{\partial \mu_{t}} d \mu d t$ gives the change in $U_{a}$ when mortality rates increase by $d \mu$ during $d t$ around $t$. A first application of Volterra derivatives to economics is Ryder and Heal (1973). 
between consumptions at two different dates whose time distance tends to zero.

Definition 2 (IES) The intertemporal elasticity of substitution at age $t$, which we denote $\sigma_{t}$, is defined by:

$$
\frac{1}{\sigma_{t}} \delta_{t} \equiv \lim _{\substack{\tau \rightarrow t \\ \tau \neq t}} \frac{-\frac{\frac{\partial^{2} E U_{a}}{\left(\partial c_{t}\right)^{2}}}{\left(\frac{\partial E U_{a}}{\partial c_{t}}\right)^{2}}+2 \frac{\frac{\partial^{2} E U_{a}}{\partial c_{t} \partial c_{\tau}}}{\frac{\partial E U_{a}}{\partial c_{t}} \frac{\partial E U_{a}}{\partial c \tau}}-\frac{\frac{\partial^{2} E U_{a}}{\left(\partial c_{\tau}\right)^{2}}}{\left(\frac{\partial E U_{a}}{\partial c_{\tau}}\right)^{2}}}{\frac{1}{c_{t} \frac{\partial E U_{a}}{\partial c_{t}}}+\frac{1}{c_{\tau} \frac{\partial E U_{a}}{\partial c_{\tau}}}}
$$

where $\delta_{t}$ is the Dirac delta function. ${ }^{10}$

The intertemporal elasticity of substitution, together with the mortality adjusted rate of time discounting are the key determinants of the marginal trade-offs involved in consumption smoothing. For example, in a perfect market environment (with actuarially fair annuities and a rate of interest $r$ ), the growth rate of the optimal path would be $(r-\operatorname{RD}(\mathbf{c}, t)) \sigma_{t}$.

With the recursive model,

$$
\sigma_{t}=-\frac{1}{c_{t}} \frac{u^{\prime}\left(c_{t}\right)-v^{\prime}\left(c_{t}\right) E U_{t}}{u^{\prime \prime}\left(c_{t}\right)-v^{\prime \prime}\left(c_{t}\right) E U_{t}}
$$

When preferences are additive or multiplicative, this formula simplifies to $\sigma_{t}=\frac{-u^{\prime}\left(c_{t}\right)}{c_{t} u^{\prime \prime}\left(c_{t}\right)}$.

The third concept of time discounting simply expresses how people trade off survival probabilities at different ages.

Definition 3 (RDLY) The rate of time discounting for life years is defined by

$$
R D L Y(\mathbf{c}, t) \equiv-\left.\frac{d}{d t} \log \left(\frac{\partial E U_{a}}{\partial s_{a}^{t}}\right)\right|_{\dot{c}_{t}=0}
$$

With the recursive model,

$$
\operatorname{RDLY}(\mathbf{c}, t)=v\left(c_{t}\right)
$$

The fourth concept, which is at the center of our analysis, requires more comments and clarifications.

\footnotetext{
${ }^{10}$ The presence of the Dirac delta function is a purely technical point related to continuous time modeling. This function appears when second order derivatives are involved. See also footnote 9 .
} 
Definition 4 (MRA) Mortality risk aversion is defined by

$$
\operatorname{MRA}(\mathbf{c}, t) \equiv \lim _{\substack{T \rightarrow t \\ T>t}}\left[-\frac{d}{d T} \log \left(\frac{\partial U_{a}(\mathbf{c}, T)}{\partial c_{t}}\right)\right]
$$

This coefficient is unaffected by an affine transformation of $U_{a}$, meaning that it represents a fundamental characteristic of individual preferences, independent of the specific representation that was chosen. If the marginal utility of life extension is decreasing in past consumption (that is if $\frac{\partial^{2} U_{a}(\mathbf{c}, T)}{\partial c_{t} \partial T}<0$ for all $T>t$ ) then $\operatorname{MRA}(\mathbf{c}, t) \geq 0$.

The terminology "mortality risk aversion" emphasizes that MRA(c, $t)$ corresponds to a coefficient of risk aversion with respect to length duration along particular (and generally not constant) consumption paths. Indeed, writing

$$
\frac{\partial U_{a}(\mathbf{c}, T)}{\partial c_{t}}=\frac{\partial U_{a}(\mathbf{c}, T)}{\partial T} \cdot\left(\frac{\partial U_{a}(\mathbf{c}, T)}{\partial c_{t}} / \frac{\partial U_{a}(\mathbf{c}, T)}{\partial T}\right)
$$

one obtains

$$
\operatorname{MRA}(\mathbf{c}, t) \equiv-\frac{\frac{\partial^{2} U_{a}(\mathbf{c}, t)}{\partial t^{2}}}{\frac{\partial U_{a}(\mathbf{c}, t)}{\partial t}}+\lim _{\substack{T \rightarrow t \\ T>t}} \frac{d}{d T} \log \left(\frac{\frac{\partial U_{a}(\mathbf{c}, T)}{\partial T}}{\frac{\partial U_{a}(\mathbf{c}, T)}{\partial c_{t}}}\right)
$$

The first term in the RHS is recognizable as a coefficient of risk aversion with respect to life duration. When consumption profiles such that

$$
\lim _{\substack{T \rightarrow t \\ T>t}} \frac{d}{d T}\left(\frac{\frac{\partial U_{a}(\mathbf{c}, T)}{\partial T}}{\frac{\partial U_{a}(\mathbf{c}, T)}{\partial c_{t}}}\right)=0
$$

are considered, $\operatorname{MRA}(\mathbf{c}, t)$ and the Arrow-Pratt coefficient are equal.

Consumption profiles that comply with (22) are characterized by the fact that the marginal rate of substitution between additional life years and consumption just before death is independent of the age at death. In particular, (22) amounts to having $u\left(c_{t}\right) e^{-\lambda t}$ constant in the additive model, and $c_{t}$ is constant with the multiplicative model. In both cases, this can be interpreted as having a constant flow of felicity (Bommier, 2006). 
The decomposition into two terms is important for understanding the origin of $\operatorname{MRA}(\mathbf{c}, t)$, but quite remarkably, with the recursive model any consumption profile leads to the following simple expression

$$
\operatorname{MRA}(\mathbf{c}, t)=\frac{v^{\prime}\left(c_{t}\right) u\left(c_{t}\right)}{u^{\prime}\left(c_{t}\right)}
$$

which depends only on local properties. Remark that $\operatorname{MRA}(\mathbf{c}, t)>(<) 0$ if $v(\cdot)$ is increasing (decreasing) and is null with the additive model.

\section{The value of statistical lives}

Subsection 4.1 defines the value of statistical lives (VSL) and relates this index with the structural parameters of the recursive model. Subsection 4.2 shows the information one can draw from empirical data to estimate preference parameters.

\subsection{VSL}

A natural concept to deal with choices involving mortality changes is the marginal rate of substitution between mortality and consumption:

Definition 5 (VSL) The value of a statistical life at age $t>a$ is defined by

$$
V S L(\mathbf{c}, t) \equiv-\left(\frac{\partial E U_{a}}{\partial \mu_{t}}\right) /\left(\frac{\partial E U_{a}}{\partial c_{t}}\right)
$$

An agent of age $t$ is ready to give up $\operatorname{VSL}(\mathbf{c}, t) \cdot d \mu \cdot d t$ in consumption to save $d \mu \cdot d t$ statistical lives. This is how we construe the term "Value of Statistical Life", although it may differ from other definitions that can be found in the economic literature. ${ }^{11}$ By derivation from (12), one obtains

$$
\operatorname{VSL}(\mathbf{c}, t)=\frac{E U_{t}}{u^{\prime}\left(c_{t}\right)-v^{\prime}\left(c_{t}\right) E U_{t}}
$$

\footnotetext{
${ }^{11}$ As discussed in Johansson (2002), various definitions of VSL have been suggested. Another popular approach is to define VSL as being the MRS between mortality rate and wealth. Then VSL not only depends on individuals' preferences but also on intertemporal constraints. This latter approach coincides with ours whenever intertemporal constraints are as those considered in Section 4.2.
} 
The following expression relates VSL to survival probabilities and discount rates.

Proposition 1 For any consumption profile

$$
V S L(\mathbf{c}, t)=\int_{t}^{+\infty} s_{t}^{\tau} \frac{u\left(c_{\tau}\right)}{u^{\prime}\left(c_{\tau}\right)} \exp \left(-\int_{t}^{\tau} \rho\left(\mathbf{c}, \tau^{\prime}\right) d \tau^{\prime}\right) d \tau
$$

with

$$
\rho\left(\mathbf{c}, \tau^{\prime}\right)=R D\left(\mathbf{c}, \tau^{\prime}\right)-M R A\left(\mathbf{c}, \tau^{\prime}\right)+\frac{1}{\sigma_{\tau^{\prime}}} \frac{\dot{c}_{\tau^{\prime}}}{c_{\tau^{\prime}}}
$$

Proof. See appendix.

In the additive case, with $c_{t}=c$ (a constant), this expression simplifies to

$$
\operatorname{VSL}(\mathbf{c}, t)=\frac{u(c)}{u^{\prime}(c)} \int_{t}^{+\infty} s_{t}^{\tau} e^{-\lambda(\tau-t)} d \tau
$$

This formula has been known for years and its simplicity explains its success. It is considered very convenient since, if we abstract from consumption variations, VSL is proportional to a discounted sum of life years. The relation between age and VSL is then computable from a standard life table and a discount rate. This way of accounting for age was initially introduced by Moore and Viscusi (1988) and is now used and recommended by agencies like the USA Environmental Protection Agency (EPA) and the Office of Management and Budget (OMB) for cost-benefit analyses.

Proposition 1 is associated with a minor increase in complexity. Although the generalization makes intermediate calculations more fastidious, we eventually find that the benefit of saving one statistical life among individuals of a given age is also proportional to the discounted sum of years at risk. Casually, we find that accounting for consumption variations is relatively simple, whether preferences are additive or not.

There are two notable differences between the additive and the recursive models. First, in the recursive model the mortality adjusted rate of discount RD is not constant. Instead of using a discount function $e^{-\lambda(\tau-t)}$, as in the additive case, we have to use $\exp \left(-\int_{t}^{\tau} \operatorname{RD}\left(c, \tau^{\prime}\right) d \tau^{\prime}\right)$. Actually, when we calibrate the model (Section 5), we find that the variations of RD remain 
limited until advanced ages, so this first difference can be considered as minor. The second difference is much more significant: years of life have to be discounted with the mortality adjusted rate of discount (RD) minus mortality risk aversion (MRA).

Consequently, the greater mortality risk aversion, the faster VSL declines as a function of age. This is fairly intuitive: a risk averse agent is willing to pay more to avoid the chance of a major loss. In terms of mortality, a loss would be an early death. The additive model, which disregards mortality risk aversion, may underestimate the speed at which VSL declines with age. The bias is estimated and confirmed in Section 5 .

\subsection{Wage-risk tradeoff}

The revealed preferences argument can be invoked to show how occupational choices provide information about utility functions. Assume that, at all ages, an individual has to choose between jobs that differ with respect to wage and instantaneous fatality risk. Let $\mu_{t}^{0}$ be the exogenous baseline mortality rate at age $t$. For an extra instantaneous mortality $\mu_{t}$ (total mortality being $\left.\mu_{t}^{0}+\mu_{t}\right)$, the wage is denoted by $w\left(t, \mu_{t}\right)$. Labor income can be used for consumption or savings. We denote by $\mathbf{k}=\left(k_{t}\right)_{t \geq 0}$ the age-specific saving profile defined by

$$
k_{t} \equiv w\left(t, \mu_{t}\right)-c_{t}
$$

For our purpose, we do not need to fully specify the lifetime budget constraints that are related to the intertemporal markets and their possible imperfections. We will simply assume that these constraints (possibly infinitely many) only bear on the function $\mathbf{k}$ and that each of them is Volterra differentiable. We denote the set of constraints by $\mathcal{K}$.

We may think of different kinds of constraints. With non storable commodities and no intertemporal markets, $k_{t}=0$ for all $t$. Another possibility would be a single constraint of the form $\int_{0}^{\infty} k_{t} h_{t} e^{-r t} d t=0$ with $r$ being the rate of interest and $\mathbf{h}=\left(h_{t}\right)_{t \geq 0}$ an exogenous function. This includes the important case of intertemporal markets, in particular life annu- 
ities. ${ }^{12}$ We could also imagine that the constraints $\mathcal{K}$ have the form $\int_{0}^{t} k_{\tau} e^{-r \tau} d \tau \geq 0$ for all $t$. That would be the case in a world where there is no annuity market, no borrowing and a rate of return on savings equal to $r$. More complex market imperfections can be thought of. Undoubtedly, allowing any kind of constraints on $\mathbf{k}$ leaves us with a fairly high degree of generality, although certain cases are not covered (e.g. nonlinear consumption taxes).

Using (4) and (12), we rewrite the lifetime utility function of an agent of age $a$ as

$$
E U_{a}(\mathbf{c}, \mu)=\int_{a}^{+\infty} u\left(c_{t}\right) \exp \left(-\int_{a}^{t}\left(\mu_{\tau}+\mu_{\tau}^{0}+v\left(c_{\tau}\right)\right) d \tau\right) d t
$$

A rational agent solves the maximization program

$$
\max _{\mu, \mathbf{c}} E U_{a}(\mathbf{c}, \mu) \text { s.t. } \mathcal{K} \text {. }
$$

The derivative $w_{\mu}(t, \mu)=\frac{\partial w(t, \mu)}{\partial \mu}$ is the "wage-risk tradeoff." Even without an explicit formulation of the constraints $\mathcal{K}$, we can show that at the optimal choice the wage risk tradeoff and the VSL are equal. Indeed, differentiating (29), for all $t, \tau$, we have

$$
\left(\frac{\partial}{\partial \mu_{t}}+w_{\mu}\left(t, \mu_{t}\right) \frac{\partial}{\partial c_{t}}\right) k_{\tau}=0
$$

Let $\mathbf{c}^{*}$ and $\mu^{*}$ denote the optimal consumption and mortality paths. As we assumed that all constraints can be written as functions of $\mathbf{k}$, the first order conditions ensure that for all $t$, utility cannot be improved without violating the constraints. Thus, because of (32), it must be the case that at the optimum

$$
\left(\frac{\partial}{\partial \mu_{t}}+w_{\mu}\left(t, \mu_{t}\right) \frac{\partial}{\partial c_{t}}\right) E U_{a}=0
$$

\footnotetext{
${ }^{12}$ To be more specific, exogenously priced life annuities are considered. Endogenous prices would mean that prices change as the consumer changes his mortality e.g. via activity choice. This case is not included here; if $\mathbf{h}$ were equal to the (endogenous) survival function, as with perfect intertemporal markets, the VSL at age $a$ would be reduced by the wealth held at age $a$. Quantitatively speaking, the correction is minor (average wealth is typically much lower than the VSL).
} 
Therefore:

$$
w_{\mu}\left(t, \mu_{t}^{*}\right)=-\left(\frac{\partial E U_{a}\left(\mathbf{c}^{*}, \mu^{*}\right)}{\partial \mu_{t}}\right) /\left(\frac{\partial E U_{a}\left(\mathbf{c}^{*}, \mu^{*}\right)}{\partial c_{t}}\right)=\operatorname{VSL}\left(\mathbf{c}^{*}, t\right) .
$$

The observation of the wage-risk tradeoff reveals VSL and makes the calibration of the utility function possible. Compared to similar results, the strength of the latter equation is that it is established without assuming complete markets.

\section{$5 \quad$ Data fitting}

\subsection{Method}

A hedonic regression fits the envelope of the choices made by the workers in the sample (Viscusi and Aldy 2008). Since the envelope is tangent to individual indifference curves, the prediction based on the hedonic regression for a vector of individual characteristics can be interpreted as the VSL for the corresponding worker. We base the calculations on this fundamental observation.

Several recent contributions estimated the relation between age and VSL from hedonic regressions and provided contrasting results (see discussion in Section 2). As an illustration, we use the result of one of them (Aldy and Viscusi, 2003, henceforth A\&V)) to calibrate our model. By doing so, we do not claim to provide undisputable estimates of the true preference parameters since they are conditional on the particular empirical age-VSL relationship we employ. Nevertheless, we comply with the objective of the paper: showing that relaxing additivity parsimoniously can significantly improve the ability of the structural model to fit the data. ${ }^{13}$ The consequences for policy recommendations are far from trivial.

\footnotetext{
${ }^{13}$ Using one of the regressions in Viscusi and Aldy (2008) is an alternative. The qualitative results they show are similar (inverted-U-shaped relationship between age and VSL with similar rates of growth), but they suggest an overall higher level of the VSL. A consensus on the ideal database and estimates is premature, and different readers may have different views, as we experienced.
} 
We use the parameters given by $\mathrm{A} \& \mathrm{~V}$ in their Table 4 :

$$
w_{\mu}^{A V}(t)=-1.92 \times 10^{7}+1.88 \times 10^{6} t-4.54 \times 10^{4} t^{2}+335.24 t^{3}
$$

where $t \in[18,62]$, expresses the individual's age in years, and $w_{\mu}$ the yearly wage in 1996 Dollars. The calibration strategy we pursue involves searching the parameters of the recursive model that best fit equation (35). ${ }^{14}$

In order to calibrate the model, we also need the age-specific consumption profile $\mathbf{c}^{*}$, which is not available in the dataset used by $\mathrm{A} \& \mathrm{~V}$. The optimal consumption profile cannot be deduced from the theoretical model without specification of the constraints $\mathcal{K}$, on which we have limited knowledge. Rather than posing specific constraints, we approximated $\mathbf{c}^{*}$ with a smoothed version of the age specific individual consumption profile reported in Lee and Tuljapurkar (1997) (see Figure 1 for the original estimates and the smoothed profile that we use). ${ }^{15}$

\subsection{Goodness of fit}

The first question that we may address is whether we can reproduce (35) with the standard additive model (namely, $v=\lambda=$ Constant and $u(c)=\frac{c^{1-\gamma}}{1-\gamma}-u_{0}$ for some constants $u_{0}$ and $\gamma$ ). The answer is positive, but with very implausible parameters. Indeed the distance minimizing discount rate is $-8.1 \%$, which explains $94 \%$ of the age-related variance in equation (35). Had we constrained the rate of discount to be greater than or equal to $3 \%$ (to approach values that are considered as reasonable), we would have at best explained $58 \%$ of the age-related variance.

At this point it is legitimate to wonder whether this poor fit is due to the fact that we only considered isoelastic instantaneous utility functions, or more fundamentally to the additive separability. We relax each of these assumptions in turn.

\footnotetext{
${ }^{14}$ As we use consumption data from a different source, we search the best fit for ages ranging from 20 to 60.

${ }^{15}$ Lee and Tuljapurkar (1997) is one the few recent studies that provide individual (not household) agespecific consumption profiles.
} 
If we simply require $u$ to be increasing and concave rather than isoelastic, we can obviously improve the fit. By considering rates of discount greater than or equal to $3 \%$, we can now explain $79 \%$ of the age-related variance. The gain in explanatory power might seem significant but, in fact, it is quite disappointing when we recall that we added an infinity of degrees of freedom to the model ( $u$ is now nonparametric). This control stage adds weight to our view that structure (additive/nonadditive) matters much more that specification (isoelastic/nonparametric), which we now illustrate.

In fact, keeping $u$ isoelastic but in the recursive form appears to be a much more efficient way to improve the predictive power of the model. We explored the case where $u(c)=$ $\frac{c^{1-\gamma}}{1-\gamma}-u_{0}$ and $v=\lambda+\beta u$; compared to the standard additive model $(\beta=0)$, this structure requires only one additional degree of freedom. Moreover it encompasses the multiplicative model (obtained when $\lambda=0$ ) described in Subsection 3.3, which has the same number of degrees of freedom as the standard additive model. In Figure 2, we report the minimum distance (the sum of squares) between the theoretical predictions and the empirical estimates, the survival weighted average $\mathrm{RD}$ being constrained to take particular values given on the horizontal axis. The results obtained with the additive and the multiplicative models are also reported. The distance on the vertical axis has been normalized so that the distance between the empirical VSL and its mean equals 1.

Opting for the recursive model dramatically increases the capacity of the theory to reproduce empirical VSL. Even if we constrain the mortality adjusted rate of discount to take reasonable positive values we still obtain an excellent fit. We can constrain the survivalweighted average RD to take any value between 3 and $7 \%$, and still explain more than $95 \%$ of age-related variability of the wage-risk tradeoff. This is much better than the additive model which only explains from 42 to $58 \%$ thereof. Table 1 reports the model's performance (variance explained and parameters) for a range of discount factors. Figure 3 illustrates the fits obtained when the average mortality adjusted rate of discount is constrained to equal $3 \%$ in both models. Interestingly enough, one can see from Table 1 or Figure 2 that when $\mathrm{RD}$ is constrained to plausible positive values, the multiplicative model does a much better 
job than the additive one, with the same number of degrees of freedom. Therefore even if one is reluctant to increase the complexity of the model, a significant gain is obtained.

\begin{tabular}{l|ccc|ccc|ccc}
\hline \hline Model & \multicolumn{2}{|c|}{ Additive $(\beta=0)$} & \multicolumn{3}{c|}{ Recursive } & \multicolumn{2}{c}{ Multiplicative $(\lambda=0)$} \\
\hline \hline & \multicolumn{3}{|c|}{$\mathrm{RD}$} & \multicolumn{3}{c|}{ Average RD } & \multicolumn{3}{c}{ Average RD } \\
& $3 \%$ & $5 \%$ & $7 \%$ & $3 \%$ & $5 \%$ & $7 \%$ & $3 \%$ & $5 \%$ & $7 \%$ \\
\hline \hline \multirow{2}{*}{ Var. explained } & $58 \%$ & $49 \%$ & $46 \%$ & $96 \%$ & $96 \%$ & $95 \%$ & $95 \%$ & $96 \%$ & $89 \%$ \\
$\widehat{\gamma}$ & 0.22 & $0.0^{*}$ & $0.0^{*}$ & 4.15 & 3.25 & 2.65 & 3.70 & 3.77 & 3.56 \\
$\widehat{\lambda}$ & $3 \%$ & $5 \%$ & $7 \%$ & $-0.04 \%$ & $0.07 \%$ & $0.15 \%$ & 0 & 0 & 0 \\
$u_{0} /\left(\frac{\bar{c}^{1-\gamma}}{1-\gamma}\right) \quad 16$ & -7.51 & -13.7 & -17.8 & 5.46 & 4.51 & 3.65 & 5.52 & 4.47 & 3.58 \\
Average MRA & 0 & 0 & 0 & $8.9 \%$ & $9.6 \%$ & $10.7 \%$ & $8.3 \%$ & $10.4 \%$ & $12.0 \%$ \\
Average RDLY & $3 \%$ & $5 \%$ & $7 \%$ & $8.3 \%$ & $9.3 \%$ & $10.5 \%$ & $7.9 \%$ & $9.7 \%$ & $11.1 \%$ \\
\hline \hline
\end{tabular}

*The elasticity of substitution is constrained to be non-negative.

Table 1: Calibration and performance.

\subsection{Evaluated parameters}

For the recursive model, as apparent in Figure 2, the curve representing the distance between predicted and actual values exhibits a flat shape around the minimum; in practice this means that the combination of parameters that optimally fit the data is difficult to state. The observation of the relation between age and VSL may not suffice to calibrate all the parameters of the model with precision.

This is not surprising given the theoretical results provided in Section 4. From equation (26) we know that what matters for determining the variations of $w_{\mu}$ along the life cycle is mainly the combination of two elements: the mortality adjusted rate of discount (RD) minus mortality risk aversion (MRA). If consumption were constant along the life cycle, we would expect empirical observation of VSL to be informative about the difference between RD and MRA, and not about each of them separately. Though in our case consumption is

\footnotetext{
${ }^{16}$ The symbol $\bar{c}$ denotes the (survival weighted) average consumption.
} 
not constant, which in principle should solve the identification problem, our estimates suffer from the same kind of indeterminacy. For each value of RD we find the best value of MRA, but it is hard to tell what is the best pair of RD and MRA.

Ultimately, to discriminate more sharply between the several likely possibilities, we should integrate data on behavior patterns that go beyond the wage-risk tradeoff. One possibility would be to look at consumption smoothing behavior (in order to estimate RD from another source), but we leave that aside for lack of adequate data. Results thereafter are systematically reported for RD taking values 3,5 and $7 \%$.

\subsection{Practical consequences}

From the last two rows of Table 1, it is possible to get a first idea about the bias generated by the additive assumption. While the additive model constrains mortality risk aversion to be absent, the recursive model gives estimates that range from $8.9 \%$ to $10.7 \%$. In other words, when people discount consumption with rates of 3,5 and $7 \%$, life years in VSL should be discounted with rates of $-5.9 \%,-4.6 \%$ or $-3.7 \%$ respectively. The additive model, which imposes the same rate of discount for consumption as for life years, is likely to cause a huge bias.

Should that lead to a major shift in policy recommendations? The next section shows that RDLY gives the rate of discount to be used for estimating the welfare equivalent of a statistical life. While the additive model constrains RDLY to equal the rate of discount, the more general model shows values of RDLY that exceed those of RD by several percentage points. This means that the additive model puts too much relative weight on the elderly. We see now how large the bias can be in practice. 


\section{Welfare evaluation}

\subsection{Objective}

In order to evaluate the social benefits of mortality risk reductions, a well defined social objective is required. The utilitarian approach axiomatized by Blackorby et al (1997) involves assuming that the social planner maximizes a stationary weighted sum of individuals' utilities at birth. The social welfare function is then given by

$$
\sum_{i} e^{-\lambda_{S} b_{i}} U_{0}^{i}
$$

where the sum is taken over all individuals, $\lambda_{S}$ is the social discount rate, $b_{i}$ is the birth year of individual $i$ and $U_{0}^{i}$ is his expected utility at birth.

We use Arthur's (1981) terminology. The welfare equivalent of a statistical life for individual $i$ is defined by

$$
\mathrm{WE}(\mathbf{c}, t) \equiv-\frac{\partial U_{0}^{i}}{\partial \mu_{t}},
$$

where $\mathbf{c}$ and $\mu$ are individual $i$ 's consumption and mortality. WE has a fairly simple expression in the general case: ${ }^{17}$

$$
\mathrm{WE}(\mathbf{c}, t)=\int_{t}^{+\infty} s_{0}^{\tau} u\left(c_{\tau}\right) \exp \left(-\int_{0}^{\tau} \operatorname{RDLY}\left(\mathbf{c}, \tau^{\prime}\right) d \tau^{\prime}\right) d \tau
$$

Like the VSL, the welfare equivalent is a discounted sum of life years. With the additive model $\mathrm{RDLY}=\mathrm{RD}$, thus it is correct to use the discount rate inferred from empirical studies on consumption smoothing to estimate the welfare equivalent of a statistical life. With the recursive model, RDLY is typically greater than the rate of time preferences estimated in studies on consumption smoothing. Thus, omission of mortality risk aversion generates a

\footnotetext{
${ }^{17}$ From (4), it follows that

$$
\frac{\partial s_{a}^{\tau}}{\partial \mu_{t}}=0 \text { if } \tau<t, \text { and } \frac{\partial s_{a}^{\tau}}{\partial \mu_{t}}=-s_{a}^{\tau} \text { if } \tau \geq t .
$$
}

Differentiating (12) then gives (38). 
pro-old age bias in the welfare evaluation of mortality risk reduction.

\subsection{Methods}

We describe now the five evaluation methods for a program that we will apply in the following subsection.

Method 1: The number of lives saved. Though there is no economic support for this method, it has been frequently used in the past. EPA and OMB still recommend reporting the number of lives saved.

Method 2: Utilitarianism with the additive utility function. The benefit of a program is measured by the social welfare function (36). Individuals are assumed to have the same additive utility function, with a rate of time preference of 3,5 and $7 \%$, the other parameters being drawn from Section 5. The social rate of discount is taken equal to the individual rate of time preference.

Method 2': Aggregate WTP with additive utility function. Assumptions on individuals are the same as for method 2 . The benefit of a program is now evaluated by the sum of the individuals' willingness to pay for such a program.

Method 3: Utilitarianism with the recursive utility function. Similar to method 2 , with the recursive model as estimated in Section 5 . The average survival weighted RD and the social rate of discount are constrained to 3,5 and $7 \%$.

Method 3': Aggregate WTP with the recursive utility function. Similar to method 2 ', with the recursive model as estimated in Section 5. The average survival weighted RD and the social rate of discount are constrained to 3,5 and $7 \%$.

In principle, method 2' (respectively 3') amounts to method 2 (respectively 3) only if one presumes that the marginal social value of consumption is equal across people of different 
ages; in other words, if redistribution is perfect. In practice, since the distribution of wealth is far from ideal with respect to the social welfare function, it has been argued that aggregate willingness to pay cannot be considered as a relevant policy indicator. The issue is not specific to life saving programs but general to any cost benefit analysis (see for example the discussion in Blackorby and Donaldson 1990). In the case of mortality reduction, Pratt and Zeckhauser (1996) stressed that because of the strong heterogeneity in mortality rates, aggregating individual willingness to pay may actually be a particularly misleading indicator. Despite these shortcomings, method 2' remains the most commonly employed in the applied literature.

\subsection{Application}

To show the magnitude of distortion in the evaluation of safety programs, we consider two fictitious programs that are assumed to have the same cost. One that decreases mortality rates proportionally and another that decreases mortality rates uniformly. For example, we could think of air quality alerts ${ }^{18}$ on the one hand and of earthquake surveillance on the other.

We denote these hypothetical interventions as A and B. Policy A is characterized by a proportional reduction of mortality rates

$$
\mu_{t} \rightarrow\left(1-\varepsilon_{A}\right) \mu_{t}
$$

and policy B by a uniform reduction of mortality rates

$$
\mu_{t} \rightarrow \mu_{t}-\varepsilon_{B}
$$

where $\varepsilon_{A}$ and $\varepsilon_{B}$ are positive constants. We take the age structure of the population and the baseline mortality rates observed in the USA in 1999. We also assume that A saves twice

\footnotetext{
${ }^{18}$ Assuming a marginal impact of air pollution proportional to baseline mortality seems reasonable to epidemiologists (Pope et al. 1995).
} 
as many (statistical) lives as B. Policy A is mostly effective for older people (and babies) while policy B saves lives uniformly. Figure 4 shows the age distribution of lives saved (it has been scaled so that A saves 2000 statistical lives while B saves only 1000). We assume that the consumption profile is $\mathbf{c}^{*}$ (see Subsection 5.1), for ages above 20. For ages below 20, and especially for babies and children, the assumption that preferences are independent of age becomes problematic. The low levels of consumption that are typically observed in the very first years of life would then imply very high marginal utility of consumption, and therefore very low values of statistical lives. This is hard to buy. To circumvent this difficulty, we maintain the assumption that preferences are independent of age and assume that consumption is the same between birth and 20. Of course this option is arbitrary, one of its merits being that most of the difference between $\mathrm{A}$ and $\mathrm{B}$ is based on effects on the adults, for which estimates are more reliable.

Intuitively, it is not very clear whether A or B should be preferred. On the one hand A saves more lives. On the other hand B saves younger people, who still have many years of life before them. We use the above five types of benefit evaluation.

The results are summarized in Table 2. By assumption, A is twice as efficient as B from the viewpoint of method 1 . The additive model in methods 2 and 2' provides an age-adjusted value of a statistical life, so the conclusion is different. Methods 2 and 2' predict that the benefits of $\mathrm{A}$ and $\mathrm{B}$ are of about the same size. The fact that $\mathrm{B}$ saves less lives than $\mathrm{A}$ is approximately compensated by the fact that it saves younger people. The question now is whether this age adjustment and this conclusion are correct. Methods 3 and 3' suggest that they are not. With the recursive model, the benefits of B appear to be much greater than those of $\mathrm{A}$. The correction related to the introduction of mortality risk aversion is anything but negligible. Passing from the additive model to the nonadditive one is a bigger step than passing from the traditional method (number of lives saved) to the additive model. ${ }^{19}$

\footnotetext{
${ }^{19}$ We could also define two additional methods that parallel methods 2 and 2 ' but make use of the multiplicative model. However, as it happens that the recursive model estimated in Section 5 is practically multiplicative, the results are very close to those obtained with methods 3 and 3 '.
} 


\begin{tabular}{l|ccc}
\hline \hline \multirow{2}{*}{ Method for benefit evaluation } & \multicolumn{3}{|c}{ Discount rate } \\
\cline { 2 - 4 } & $3 \%$ & $5 \%$ & $7 \%$ \\
\hline 1. Number of lives saved & 0.5 & 0.5 & 0.5 \\
2. Utilitarianism with additive utility & 1.11 & 0.97 & 0.88 \\
3. Utilitarianism with recursive utility & 3.23 & 2.64 & 2.18 \\
2'. Aggregate WTP with additive utility & 0.94 & 0.82 & 0.75 \\
3'. Aggregate WTP with recursive utility & 1.95 & 1.75 & 1.72 \\
\hline \hline
\end{tabular}

Table 2: Benefits of B/Benefits of A.

EPA guidelines advise performing sensitivity analysis by calculating the results of both methods 1 and 2'. As the results of method 2' are known to depend on the rate of discount, about which there is no general agreement, they advise reporting the results for different rates lying in the 3-7\% interval, in order to provide a reasonable confidence interval. Unfortunately, the additive model is so restrictive that the truth may be way outside this interval. The methods currently used by EPA and OMB (and indirectly by policymakers) are likely to be significantly distorted in favor of the old.

\section{Conclusion}

Most economists would agree that predicting saving behavior under the assumption of risk neutrality would make little sense. They would also vehemently criticize a fund manager who decides to "optimize" investment under the assumption that members are risk neutral.

However, the economic literature on the value of a statistical life has endorsed a similar choice. It focused on a specification that paid little attention to the fact that mortality makes our life akin to an extraordinary lottery. Is it reasonable to assume that individuals are risk neutral with respect to length of life? And to evaluate life saving programs under this assumption?

These questions have been addressed in this paper. On the theoretical side, the story is clear. Mortality risk aversion makes individual willingness to pay for mortality risk reduction 
decline more rapidly with age. Although intermediate calculations are sometimes fastidious, we eventually found that accounting for mortality risk aversion is fairly simple. Just like with the standard additive model, estimating VSL and welfare benefits associated to mortality risk reduction simply involves computing weighted sums of life-years saved. The rates of discount to be used must however account for both time preferences and mortality risk aversion.

The key issue is therefore to estimate mortality risk aversion. The difficulty of the task should not be underestimated. Since Arrow's (1971) and Pratt's (1964) seminal contributions, about 40 years have passed and a number of empirical studies tried to measure risk aversion with respect to lotteries on wealth. No consensus has emerged. There is no reason to believe that preferences with respect to lotteries on the length of life will be easier to assess. It would be excessively optimistic to expect that a single study could provide a robust estimate of mortality risk aversion. This should be rather seen as a long term objective that will probably require the collection of specific data.

However, in order to clarify the ideas at stake, we used results from a recent empirical study on the relation between VSL and age to estimate plausible values of mortality risk aversion. The theoretical extension neatly improved the quality of fit. We found that this index of risk aversion is likely to be positive and greater than the rate of time discounting. In other words, accounting for mortality risk aversion may even be more important than accounting for time preferences.

The contrast between our findings and the dominant economic approach is striking. While the notion of time preferences has been pointed out as being a critical element to estimate the value of a statistical life, the standard method simply rules out mortality risk aversion. It seems that "the paradigm of optimizing a simple functional form" (to take Rubinstein's 2003 words) has led economists to ignore a key ingredient of individual preferences. The consequence is that cost-benefit analysis produced for the allocation of public money across life saving programs is likely to be strongly distorted. 


\section{References}

[1] Alberini, A., M. Cropper, A. Krupnick, and N. Simon, 2004, "Does the Value of a Statistical Life Vary with Age and Health Status? Evidence from the U.S. and Canada." Journal of Environmental Economics and Management, 48(1): 769-792.

[2] Aldy, J.E. and W.K. Viscusi, 2003, Age Variations in Workers' Value of Statistical Life. NBER WP 10199.

[3] Aldy, J.E. and W.K. Viscusi, 2008, "Adjusting the Value of a Statistical Life for Age and Cohort Effects." Review of Economics and Statistics, 90(3): 573-581.

[4] Arrow, K.J., 1971, Essays in the Theory of Risk Bearing. Chicago: Markham.

[5] Arthur, W.B., 1981, "The Economics of Risks to Life." American Economic Review, 71(1): $54-64$.

[6] Blackorby, C., W. Bossert and D. Donaldson, 1997, "Birth-Date Dependent Population Ethics: Critical-Level Principles." Journal of Economic Theory, 77(2): 260-284.

[7] Blackorby, C. and D. Donaldson, 1990, "A Review Article: The Case against the Use of the Sum of Compensating Variations in Cost-Benefit Analysis." Canadian Journal of Economics, 23(3): 471-494.

[8] Bommier, A., 2006, "Uncertain Lifetime and Inter-temporal Choice: Risk Aversion as a Rationale for Time Discounting." International Economic Review, 47(4): 1223-1246.

[9] Bommier, A., 2005, Life Cycle Theory for Human Beings. Working Paper, University of Toulouse.

[10] Broome, J., 1993, "Qalys.” Journal of Public Economics, 50(2): 149-176.

[11] Browning, M., 1991, "A Simple Nonadditive Preference Structure for Models of Household Behavior over Time." Journal of Political Economy, 99(3): 607-637. 
[12] Carrasco, R., J. Labeaga and J. López-Salido, 2005, "Consumption and Habits: Evidence from Panel Data." Economic Journal, 115(500): 144-165.

[13] Deaton, A., 1974, "A Reconsideration of the Empirical Implications of Additive Preferences." Economic Journal, 84(334): 338-348.

[14] Deaton, A., 1992, Understanding Consumption. Oxford University Press.

[15] Eeckhoudt, L.R. and J.K. Hammitt, 2004, "Does risk aversion increase the value of mortality risk?. " Journal of Environmental Economics and Management, 47(1): 13-29.

[16] Epstein, L.G., 1983, "Stationary Cardinal Utility and Optimal Growth Under Uncertainty." Journal of Economic Theory, 31: 133-52.

[17] Epstein, L.G., 1987, “A Simple Dynamic General Equilibrium Model.” Journal of Economic Theory, 41: 68-95.

[18] Epstein, L.G. and S.E. Zin, 1991, "Substitution, Risk Aversion, and the Temporal Behavior of Consumption and Asset Returns: An Empirical Analysis." Journal of Political Economy, 99(2): 263-286.

[19] Ehrlich, I. and Y. Yin, 2005, "Explaining Diversities in Age-Specific Life Expectancies and Values of Life Saving: A Numerical Analysis." Journal of Risk and Uncertainty, 31(2): 129-162.

[20] Hall, R.E. and C.I. Jones, 2007, "The Value of Life and the Rise in Health Spending." Quarterly Journal of Economics, 122(1): 39-72.

[21] Hayashi, F., 1985, "The Effect of Liquidity Constraints on Consumption: A Cross-Sectional Analysis." Quarterly Journal of Economics, 100(1): 183-206.

[22] Johansson, P.O., 2002, "On the Definition and Age-Dependency of the Value of a Statistical Life." Journal of Risk and Uncertainty, 25(3): 251-263.

[23] Kihlstrom, R.E. and L.J. Mirman, 1974,. "Risk Aversion with Many Commodities." Journal of Economic Theory, 8(3): 361-88. 
[24] Kaplow, L., 2005, "The Value of a Statistical Life and the Coefficient of Relative Risk Aversion." Journal of Risk and Uncertainty, 31(1), 23-34.

[25] Kniesner, T.J., W.K. Viscusi, and J.P. Ziliak, 2006, "Life-Cycle Consumption and the AgeAdjusted Value of Life." Contributions to Economic Analysis \& Policy: 5(1):Article 4.

[26] Lee, R.D. and S. Tuljapurkar, 1997, "Economic Consequences of Aging for Populations and Individuals Death and Taxes: Longer Life, Consumption, and Social Security." Demography, 34(1): $67-81$.

[27] McFadden, D., 1963, "Constant Elasticity of Substitution Production Functions." Review of Economic Studies, 30(2): 73-83

[28] Moore, M.J. and W.K. Viscusi, 1988, "The Quantity Adjusted Value of Life." Economic Inquiry, 26: 368-388.

[29] Muellbauer, J., 1988, "Habits, Rationality and Myopia in the Life-cycle Consumption Function." Annales d'Economie et de Statistique, 9: 47-70.

[30] Murphy, K.M. and R.H. Topel, 2006, "The Value of Health and Longevity.” Journal of Political Economy, 114(5): 871-904.

[31] Pope, C.A. 3rd, M.J. Thun, M.M. Namboodiri, D.W. Dockery, J.S. Evans, F.E. Speizer and C.W. Jr Heath, 1995, "Particulate Air Pollution as a Predictor of Mortality in a Prospective Study of U.S. Adults." American Journal of Respiratory and Critical Care Medicine, 151(3 Pt 1): 669-74.

[32] Pratt, J., 1964, "Risk Aversion in the Small and in the Large." Econometrica, 32: 122-136.

[33] Pratt, J.W. and R.J. Zeckhauser, 1996, "Willingness to Pay and the Distribution of Risk and Wealth." Journal of Political Economy, 104(4): 747-763.

[34] Richard, S.F., 1975, "Multivariate Risk Aversion, Utility Independence and Separable Utility Functions." Management Science, 22(1): 12-21. 
[35] Rosen, S., 1988, "The Value of Changes in Life Expectancy." Journal of Risk and Uncertainty, 1: $285-304$.

[36] Rubinstein, A., 2003, “'Economics and Psychology'? The Case of Hyperbolic Discounting." International Economic Review, 44: 1207-1216.

[37] Ryder, H.E. Jr. and G.M. Heal, 1973, "Optimum Growth with Intertemporally Dependent Preferences." Review of Economic Studies, 40(1): 1-33.

[38] Shepard, D.S. and R.J. Zeckhauser, 1984, "Survival versus Consumption." Management Science, 30(4): 423-439.

[39] Smith, V.K., M.F. Evans, H. Kim, and D.H. Taylor, 2004, "Do the Near-Elderly Value Mortality Risks Differently?" Review of Economics and Statistics, 86(1): 423-429.

[40] Uzawa, H., 1969, "Time Preference and the Penrose Effect in a Two-Class Model of Economic Growth." Journal of Political Economy, 77(4): 628-652.

[41] Viscusi, W.K. and J.E. Aldy, 2003, "The Value of a Statistical Life: A Critical Review of Market Estimates throughout the World." Journal of Risk and Uncertainty, 27(1): 5-76.

[42] Viscusi, W.K. and J.E. Aldy, 2007, "Labor market estimates of the senior discount for the value of statistical life." Journal of Environmental Economics and Management, 53(3): 377-392.

[43] Villeneuve, B., 2000, Life Insurance. Handbook of Insurance (chapter 27), G. Dionne (ed.). Kluwer Academic Publishers.

[44] Yaari, M.E., 1965, "Uncertain Lifetime, Life Insurance, and the Theory of the Consumer?" Review of Economic Studies, 32(2): 137-150. 


\section{A Proof of Proposition 1}

In the proof, VSL stands for $\operatorname{VSL}(\mathbf{c}, t)$ and $\mathrm{RD}$ for $\mathrm{RD}(\mathbf{c}, t)$. We start from $(25)$ and we use the fact that

$$
\frac{d E U_{t}}{d t}=\left(\mu_{t}+v\left(c_{t}\right)\right) E U_{t}-u\left(c_{t}\right)
$$

to compute

$$
\begin{aligned}
& \frac{d}{d t} \log \mathrm{VSL}= \\
& \quad \mu_{t}+v\left(c_{t}\right)-\frac{u\left(c_{t}\right)}{E U_{t}}-\frac{u^{\prime \prime}\left(c_{t}\right)-v^{\prime \prime}\left(c_{t}\right) E U_{t}}{u^{\prime}\left(c_{t}\right)-v^{\prime}\left(c_{t}\right) E U_{t}} c_{t}+v^{\prime}\left(c_{t}\right) \frac{\left(\mu_{t}+v\left(c_{t}\right)\right) E U_{t}-u\left(c_{t}\right)}{u^{\prime}\left(c_{t}\right)-v^{\prime}\left(c_{t}\right) E U_{t}}
\end{aligned}
$$

Using (14) and (16), we get

$$
\frac{d}{d t} \log \mathrm{VSL}=\mu_{t}+\frac{1}{\sigma_{t}} \frac{\dot{c}_{t}}{c_{t}}+\mathrm{RD}-\frac{u\left(c_{t}\right)}{E U_{t}} .
$$

From (25), we obtain

$$
E U_{t}=\frac{u^{\prime}\left(c_{t}\right) \mathrm{VSL}}{1+v^{\prime}\left(c_{t}\right) \mathrm{VSL}}
$$

thus

$$
\frac{u\left(c_{t}\right)}{E U_{t}}=\frac{u\left(c_{t}\right)\left(1+v^{\prime}\left(c_{t}\right) \mathrm{VSL}\right)}{u^{\prime}\left(c_{t}\right) \mathrm{VSL}}=\frac{u\left(c_{t}\right) v^{\prime}\left(c_{t}\right)}{u^{\prime}\left(c_{t}\right)}+\frac{u\left(c_{t}\right)}{u^{\prime}\left(c_{t}\right)} \frac{1}{\mathrm{VSL}} .
$$

Combining (45) with (43) yields

$$
\frac{d}{d t} \log \mathrm{VSL}=\mu_{t}+\frac{1}{\sigma_{t}} \frac{\dot{c}_{t}}{c_{t}}+\mathrm{RD}-\frac{u\left(c_{t}\right) v^{\prime}\left(c_{t}\right)}{u^{\prime}\left(c_{t}\right)}-\frac{u\left(c_{t}\right)}{u^{\prime}\left(c_{t}\right)} \frac{1}{\mathrm{VSL}},
$$

i.e.

$$
\frac{d \mathrm{VSL}}{d t}=\left(\mu_{t}+\mathrm{RD}-\frac{u\left(c_{t}\right) v^{\prime}\left(c_{t}\right)}{u^{\prime}\left(c_{t}\right)}+\frac{1}{\sigma_{t}} \frac{\dot{c}_{t}}{c_{t}}\right) \mathrm{VSL}-\frac{u\left(c_{t}\right)}{u^{\prime}\left(c_{t}\right)}
$$

We show now in three steps that

$$
\operatorname{VSL}(\mathbf{c}, t)=\int_{t}^{+\infty} s_{t}^{\tau} \frac{u\left(c_{\tau}\right)}{u^{\prime}\left(c_{\tau}\right)} \exp \left(-\int_{t}^{\tau} \rho\left(\mathbf{c}, \tau^{\prime}\right) d \tau^{\prime}\right) d \tau
$$


with

$$
\rho\left(\mathbf{c}, \tau^{\prime}\right)=\operatorname{RD}\left(\mathbf{c}, \tau^{\prime}\right)-\frac{u\left(c_{\tau}\right) v^{\prime}\left(c_{\tau}\right)}{u^{\prime}\left(c_{\tau}\right)}+\frac{1}{\sigma_{\tau}} \frac{\dot{c}_{\tau}}{c_{\tau}} .
$$

Step 1. It is easy to see that the RHS of (48), if it converges, is a solution to the ODE (47).

Step 2. Remark that $E U_{t}>0$. Indeed, a natural assumption is that the marginal value of life years, which is proportional to $u$, is positive, and $u>0$ implies $E U_{t}>0$.

Given Assumptions 1 and 2, $E U_{t}$ tends to zero as $t$ tends to infinity. This and (25) imply that VSL $\rightarrow 0$ as $t \rightarrow+\infty$. We can also conclude from this, (14) and $E U_{t}>0$, that $\mathrm{RD}$ is bounded below in the long run. Consequently, $\rho(\mathbf{c}, t) \rightarrow+\infty$ as $t \rightarrow+\infty$. This implies that the RHS of $(48) \rightarrow 0$ as $t \rightarrow+\infty$. VSL and the RHS of (48) have therefore the same limit when $t \rightarrow+\infty$.

Step 3. The ODE (47) being linear, if we denote by $y$ the difference between the VSL and the RHS of (48), we have

$$
y^{\prime}=\rho(\mathbf{c}, t) y
$$

Given that $\rho(\mathbf{c}, t) \rightarrow+\infty$ as $t \rightarrow+\infty, y$ goes to infinity when $t \rightarrow+\infty$ if it's not null. This fact, combined with the result on limits (step 2), proves that (48) is true. 
Figure 2: Goodness of fit

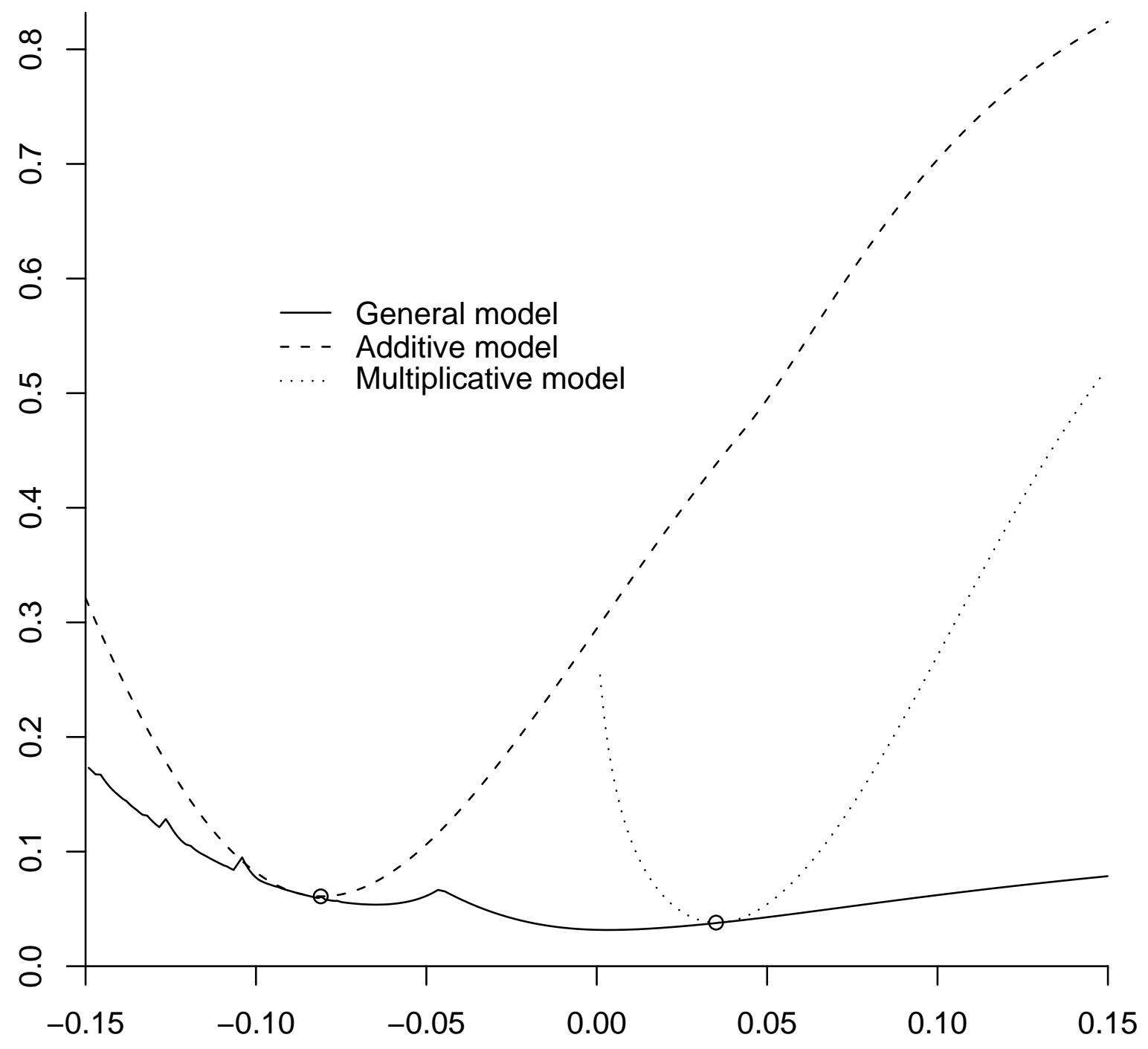

Average RD 
Figure 3: Age dependent value of a statistical life

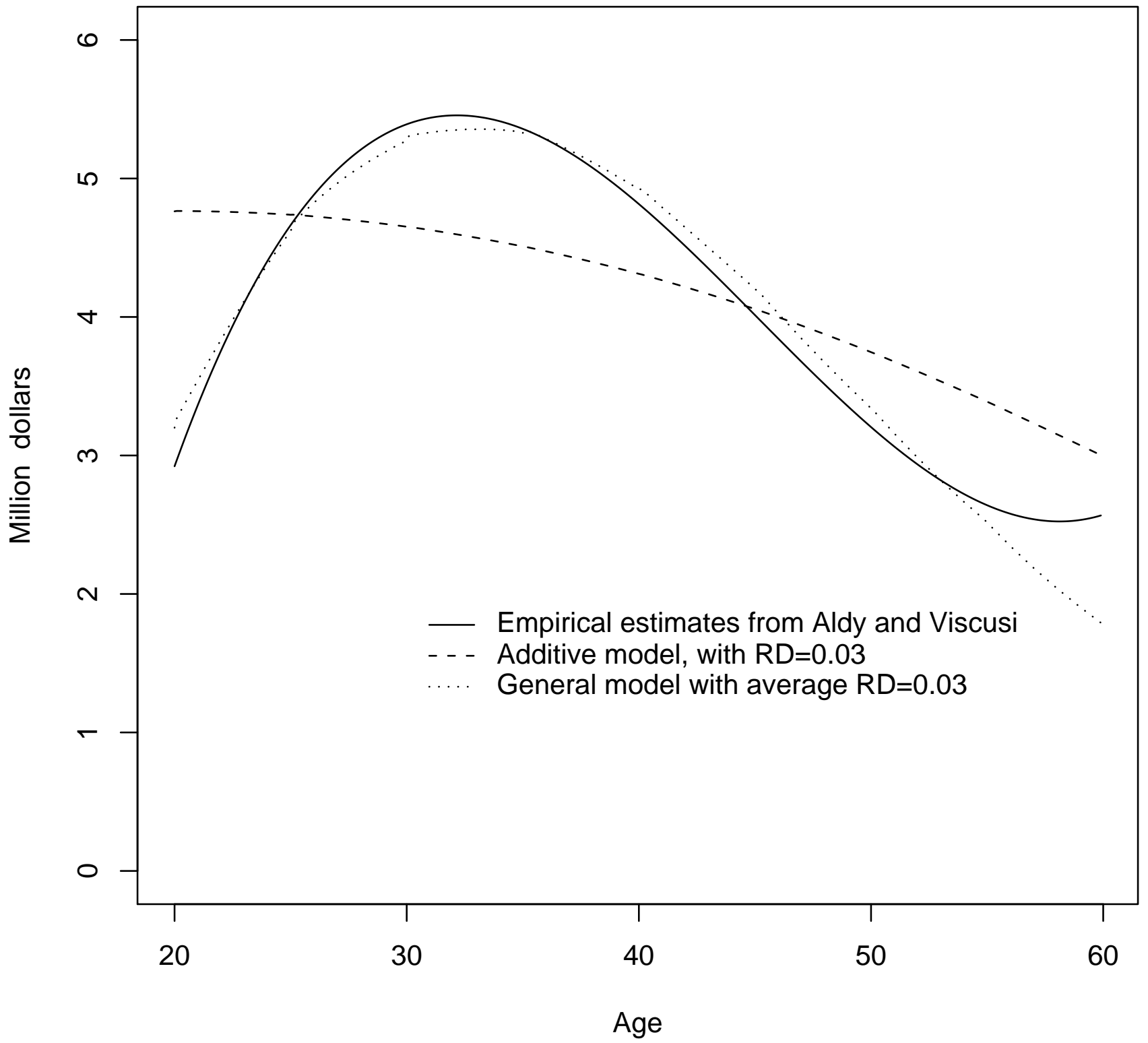


Figure 4: Distribution of lives saved

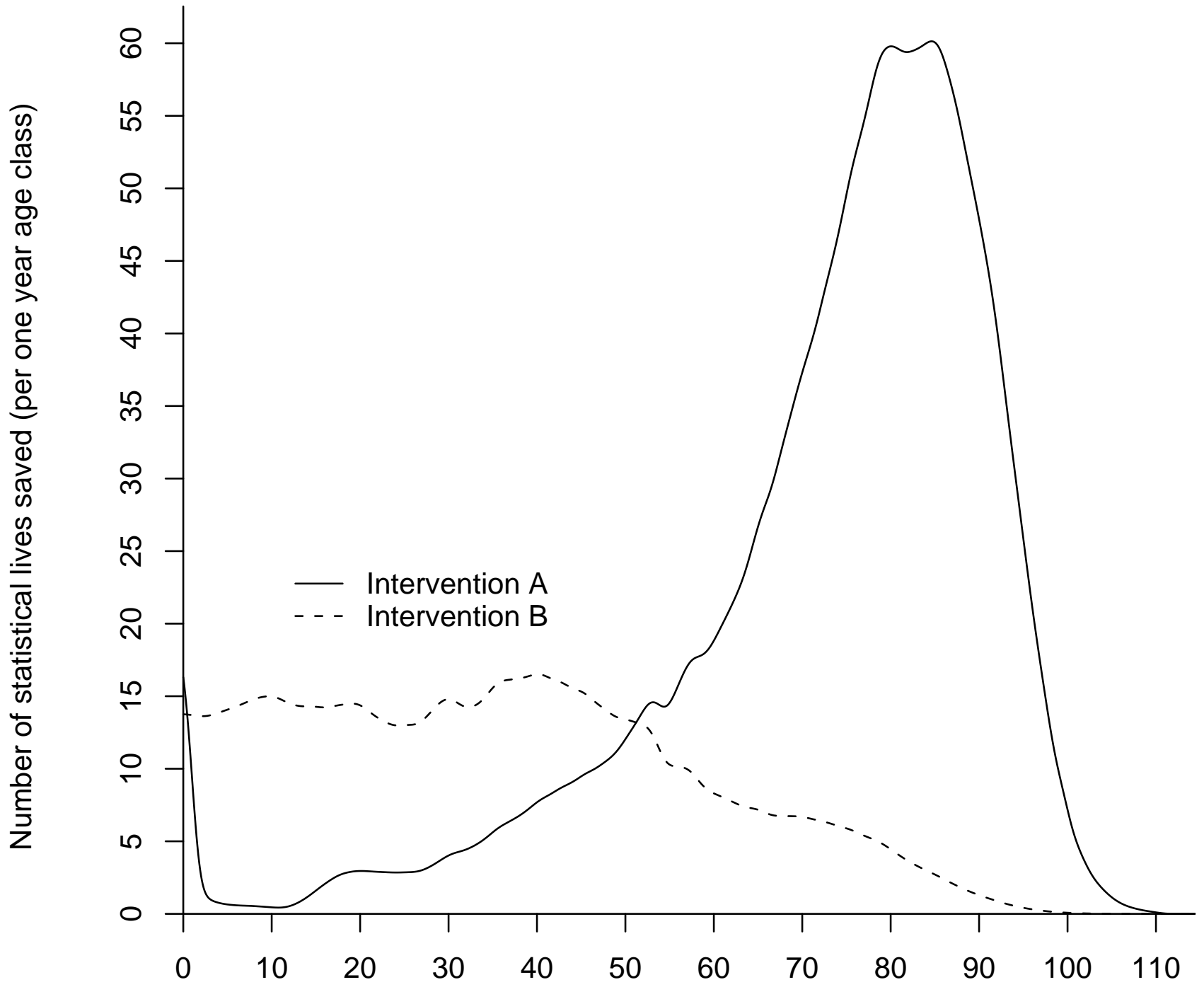

